\title{
Respuesta de cortisol salival y ansiedad precompetitiva en nadadores Salivary cortisol response and precompetitive anxiety in swimmers
}

Heriberto Antonio Pineda-Espejel, Marina Trejo, Karime Berenice García, Kristhel Judith Garza, Gustavo VázquezJiménez, Jesús René Machado-Contreras, María Esther Mejía-León, Samuel Rodríguez

Universidad Autónoma de Baja California (México)

Resumen. El objetivo de este trabajo fue medir los niveles de ansiedad precompetitiva y su relación con la secreción de cortisol medido a nivel salival dentro de una competición oficial de natación. Participaron 19 nadadores de ambos sexos, con una edad media de 12.64 años $(D T=1.65)$. La medida fisiológica incluyó cortisol salival medido con ELISA. En adición, estados psicológicos precompetitivos fueron medidos con el CSAI-2R. La recolección de datos se llevó a cabo en dos tomas, en la Toma 1 se midió el cortisol salival tres semanas antes de una competición (línea base); mientras que en la toma 2 se midió el cortisol salival 16 horas previas a la competición, y se midió la ansiedad precompetitiva. Las dos colectas se realizaron a las 16 horas. Para el análisis de los datos, la muestra se dividió en dos submuestras, una con participantes de 10 y 11 años de edad, y la otra con los de 12 a 16 años de edad. Los resultados del análisis estadístico convencional por hipótesis nula, rechazan diferencias significativas en la concentración de cortisol salival entre las dos tomas, para ambas submuestras. No obstante, el cambio mínimo relevante confirmó un cambio relevante de cortisol salival precompetitivo para la primer submuestra. Además, ambas submuestras presentan baja ansiedad precompetitiva. Las concentraciones de cortisol un día antes de la competición no correlacionaron con la ansiedad (somática y cognitiva). En conclusión, los bajos niveles de cortisol, y altos niveles de autoconfianza sugieren una respuesta psicobiológica adaptativa a la competición. Esto puede contribuir al rendimiento y resultados esperados en la competición.

Palabras clave: deporte, ansiedad cognitiva, ansiedad somática, autoconfianza, respuesta hormonal.

\begin{abstract}
The objective of this work was to measure the levels of precompetitive anxiety and its relationship with the secretion of cortisol measured at the salivary level within an official swimming competition. 19 swimmers of both sexes participated, with an average age of 12.64 years old $(\mathrm{SD}=1.65)$. The physiological measure included salivary cortisol measured with ELISA. In addition, precompetitive psychological states were measured with the CSAI-2R. Data collection was carried out at two times, in time 1 salivary cortisol was measured three weeks before a competition (baseline); while in time 2 salivary cortisol was measured 16 hours before the competition, and precompetitive anxiety was measured. The two collections were made at 16 hours. For the analysis of the data, the sample was divided into two subsamples, one with participants 10 and 11 years old, and the other with those aged 12 to 16 years. The results of the conventional statistical analysis by null hypothesis, reject significant differences in the concentration of salivary cortisol between the two moments, for both subsamples. However, the relevant minimum change confirmed a significant change of precompetitive salivary cortisol for the first subsample. In addition, both subsamples have low precompetitive anxiety. Cortisol concentrations one day before the competition did not correlate with anxiety (somatic and cognitive). In conclusion, low cortisol levels and high levels of self-confidence suggest an adaptive psychobiological response to competitions. This can contribute to the performance and expected results in the competition.
\end{abstract}

Keywords: sport, cognitive anxiety, somatic anxiety, self-confidence, hormonal response.

\section{Introducción}

El estrés atribuido a la competición deportiva puede desencadenar una serie de sentimientos emocionales en los deportistas, provocando respuestas neuroendocrinas capaces de cambiar de manera aguda o crónica la función orgánica (Gimeno, Buceta \& Llantada, 2007).

Estrés, es el proceso psicosocial que envuelve la percepción individual del desbalance entre la demanda del ambiente y la capacidad de responder a ella. Este proceso es seguido de la respuesta individual a la percepción de la demanda (Valdés, 2002); la respuesta puede ser fisiológica o psicológica. Un ejemplo de respuesta psicológica emocional es la ansiedad, que aparece cuando el deportista determina que no posee los recursos (confianza, experiencia, habilidad) para manejar una situación determinada (Valdés, 2002).

La ansiedad, como respuesta a estímulos estresantes, es un estado emocional displacentero caracterizado por el nerviosismo, preocupación y aprensión, asociados con la activación del cuerpo (Weinberg \& Gould, 2010). A menudo se

Fecha recepción: 23-04-16. Fecha de aceptación: 18-10-19 Heriberto Antonio Pineda-Espejel antonio.pineda@uabc.edu.mx manifiesta a través de respuestas individuales de falta de adaptación a escala fisiológica, comportamental y cognitiva, que obstaculizan el rendimiento.

Desde la teoría multidimensional de la ansiedad competitiva (Martens, Vealey \& Burton, 1990) se distinguen tres componentes: el primer componente es la ansiedad somática, que incluye elementos fisiológicos y afectivos de la experiencia de ansiedad, dada directamente por el grado de activación del sistema nervioso autónomo; el segundo componente es la ansiedad cognitiva, relacionada con la percepción, formación de imágenes y conceptos, el pensamiento, el juicio y la imaginación; e integra el constructo de autoconfianza como un tercer componente, que es la creencia o grado de seguridad que poseen los individuos sobre su capacidad para tener éxito en su deporte.

El deporte, al girar habitualmente en torno a ganar o perder en la competición, puede provocar ansiedad (Jaenes, 2000). En el deporte se reconoce la ansiedad precompetitiva, que es el aumento progresivo de la ansiedad a medida que se acerca una competición. Este aumento de ansiedad, aunque fisiológico, se da en el periodo que va desde la víspera de la competición hasta pocos minutos antes de ésta, permitiendo que el organismo se prepare lo mejor posible para el esfuerzo (Tamorri \& Benzi, 1990). 
Por otro lado, se distinguen exposiciones de estrés agudas y crónicas. La competición deportiva es un ejemplo de exposición aguda que activa el eje hipotalámico-pituitarioadrenal (HPA) (Loucks, Mortola, Girton \& Yen, 1989), porque la competición se caracteriza por ser una situación de evaluación social de las acciones, donde el resultado es impredecible, y en ocasiones la situación es inestable. Entonces, en anticipación a, o en respuesta a estresores psicológicos, se estimula el eje (HPA) (Gaab, Rohlede, Nater \& Ehlert, 2005). El hipotálamo integra diferentes influencias de estrés, y las repuestas del hipotálamo se reflejan vía sistema endócrino, el sistema nervioso central, y la conducta (Steinacker et al., 2000). La activación del HPAse asocia con la liberación de glucocorticoides al torrente sanguíneo, especialmente cortisol, el glucocorticoide más activo (García, Martí, Vallès, Dal-Zotto \& Armario, 2000).

El cortisol es una hormona esteroidea producida en la corteza adrenal; su influencia dentro del organismo se relaciona con el estímulo del catabolismo de las proteínas. Además, tiene un efecto antiinflamatorio, y participa en el incremento de la vasoconstricción causada por la adrenalina, preparando al cuerpo para cambios externos y adaptaciones como respuesta al estrés (Edwards \& Kurlander, 2010). Esta hormona interviene en respuestas fisiológicas y conductuales por los cambios físicos y estresores psicológicos; es decir, prepara al cuerpo para responder a una situación estresante de naturaleza física, inestable e incontrolable (Dickerson \& Kemeny, 2004), como lo es la competición deportiva.

Se ha evidenciado que los niveles de cortisol salival pueden elevarse previo a una competición como respuesta anticipatoria al estrés, preparando al organismo para afrontar dichas demandas (e.g. Filaire, Alix, Ferrand \& Verger, 2009). Algunos estudios demuestran diferencias significativas en la concentración de cortisol salival entre la línea base y el momento precompetitivo. Por ejemplo, Filaire, Maso, Sagnol, Ferrand \& Lac (2001) con judocas adultos, encontraron cambios en cortisol salival entre un día de descanso y el día de competición, sin importar el resultado de la misma (perder o ganar). Maso, Gazorla, Godemet, Michaux, Lac \& Robert (2002) con jugadores de rugby adultos, notaron un incremento significativo en cortisol salival entre el día de descanso y antes de la competición (momento precompetitivo). Lo mismo observaron Elloumi, Ounis, Tabka, Van Praagh, Michaux \& Lac (2008) en jugadores de rugby adultos, Filaire et al. (2009) y Lautenbach, Laborde, Klämpf \& Achtzehn (2015) en tenistas, y Reynoso-Sánchez et al. (2017) en jugadores de balonmano adultos.

Bateup, Booth, Shirtcliff \& Granger (2002) encontraron diferencias significativas en la concentración de cortisol salival entre la línea base (sin ser un día de descanso) y el momento precompetitivo en jugadoras de rugby adultas. Lo mismo se obtuvo en los estudios de Kivlighan, Granger \& Booth (2005) y Filaire, Portier, Onen \& Filaire (2010), el primero llevado a cabo en remeros adultos, y el segundo en tenistas adolescentes. Sin embargo, otros estudios no han encontrado diferencias significativas en los niveles de cortisol salival medidos en la línea base y el momento precompetitivo (e.g. Le Panse et al., 2012).

La secreción de cortisol junto con el nivel de ansiedad, pueden proveer información sobre estrés competitivo como lo muestran algunos estudios al correlacionar el cortisol salival y la ansiedad estado competitiva (e.g. Salvador, Suay, González-Bono \& Serrano, 2003). En golfistas adultos, Doan, Newton, Kraemer, Kwon \& Schhet (2007), encontraron correlación entre el incremento de niveles de cortisol salival y la ansiedad. Resultados similares fueron obtenidos en los estudios de Elloumi et al. (2008) en jugadores de rugby adultos, y Filaire et al. (2009) en tenistas adultos. Por su parte, Papacosta, Nassis \& Gleeson (2016) demostraron que alto nivel de ansiedad cognitiva correlaciona con altos niveles de cortisol salival en judokas adultos. En tanto que Arruda, Aoki, Paludo \& Moreira (2014) mostraron que la ansiedad somática correlacionó con el cortisol salival precompetitivo en baloncestistas adultos.

No obstante, otros estudios no encuentran correlación entre los niveles de cortisol salival y la ansiedad antes de la competición. Por ejemplo, en los estudios de Salvador et al. (2003) con judokas adultos, y de Bello (2017) con deportistas adolescentes, los niveles de cortisol salival y los puntajes de ansiedad no se correlacionaron. Del mismo modo, McKay, Selig, Carlson \& Morris (1997), Thatcher, Thatcher \& Dorling (2004), Carré, Muir, Belanger \& Putnam (2006) y Haneishi, Fry, Moore, Schilling, Li \& Fry (2007) no encontraron correlación entre cortisol salival y ansiedad somática precompetitiva.

El estudio de la ansiedad en el deporte ha tenido relevancia, debido a los cambios atencionales, la distracción aumentada, el estrechamiento del campo visual, la contracción simultánea y no deseada de músculos agonistas y antagonistas, que se generan ante elevada ansiedad, y que pueden alterar el desempeño deportivo. En suma, el estrés indicado por cortisol (medición confiable de la activación del HPA) es importante en la preparación para la competición. Particularmente en la natación, al ser un deporte individual donde se busca romper marcas de tiempo, y en su competición sólo tienen una oportunidad para dar la mejor marca y poder calificar a la etapa final, y así disputar las medallas. Por lo que la exigencia de rendimiento es máxima desde el inicio de la competencia (Ponseti, García, Cantallops \& Vidal, 2017).

La elevación en la concentración de cortisol bajo el efecto de estrés resultante de una carga física o mental por la competición, está bien demostrada en el adulto de ambos sexos; sin embargo, son pocos los estudios sobre respuesta hormonal y competición en natación, y en niños y adolescentes. Por lo anterior, el objetivo de este trabajo es medir los niveles de ansiedad precompetitiva y su relación con la secreción de cortisol medido a nivel salival dentro de una competición oficial de natación.

Nosotros esperamos que los niveles de cortisol salival sean altos en la etapa precompetitiva, en comparación con un día habitual de entrenamiento; y que los deportistas tengan alta ansiedad antes de la competición. Además, que el cortisol salival se relacione positivamente con la ansiedad precompetitiva.

\section{Método}

\section{Participantes}

Mediante muestreo por conveniencia, se obtuvo una 
muestra de 19 nadadores de ambos sexos (cuatro mujeres y 15 hombres), integrantes de un equipo de natación de la Universidad Autónoma de Baja California, quienes participaron voluntariamente en el estudio. Todos ellos contaban con experiencia en competiciones nacionales. Reportaron una edad de entre 10 y 16 años $(M=12.64$ años; $D T=1.65)$, y una experiencia deportiva media de 3.35 años $(D T=1.99)$. Entrenaban entre dos y tres horas al día, por cinco a seis días a la semana.

Dentro de los criterios de inclusión estuvieron que reportaran estar libres de drogas, y sin historial de enfermedades cardiovasculares o endócrinas; aquellos que necesitaran tomar algún medicamento durante el periodo del estudio fueron excluidos.

Con base en los rangos estimados por Salimetrics (2015), la muestra, para su análisis se dividió en dos subgrupos, uno que incluye a los participantes de entre 10 y 11 años de edad, y otro que incluye a los de 12 a 16 años de edad.

\section{Instrumentos y material}

Para medir la intensidad de ansiedad competitiva se utilizó la adaptación al español en el contexto mexicano del Inventario de Ansiedad Estado Competitiva-2 Revisado (Pineda-Espejel, López-Walle \& Tomás, 2014). Está compuesto por 17 ítems. Siete de ellos miden la intensidad de ansiedad somática (e.g. «Estoy muy inquieto»), cinco ítems miden la intensidad de ansiedad cognitiva (e.g. «Me preocupa perder»), y cinco miden la autoconfianza (e.g. «Estoy seguro de mí mismo»). Se responde en una escala Likert de 5 puntos en la cual 1 corresponde a nada y 4 corresponde a mucho. La fiabilidad (alfa de Cronbach) de las subescalas del instrumento en este estudio fue adecuada ( $\mathrm{a}=.85$ para ansiedad somática; $\mathrm{a}=.94$ para ansiedad cognitiva; $\mathrm{a}=.88$ para autoconfianza).

Para la recolección de muestra de saliva se utilizaron tubos estériles de propileno transparentes con tapón, y con capacidad de 2 ml SalivaBio Saliva Collection Aid y Cryovials, Salimetrics.

\section{Procedimiento}

Este trabajo se realizó siguiendo las directrices éticas propuestas por la Asociación Americana de Psicología (APA, American Psychological Association), de acuerdo con la Declaración de Helsinki, y bajo la aprobación del Comité local de ética médica. Previo a la recolección de datos, se contactó al entrenador del equipo de natación para solicitar su autorización y llevar a cabo el estudio. En seguida, se solicitó el consentimiento informado de participación firmado por los padres o tutores de los nadadores.

El primer día se citó a los nadadores para completar la anamnesis sobre edad, sexo, entrenamiento a la semana, y salud subjetiva por medio de una historia clínica, realizada por un profesional de la salud, para descartar procesos de inflamación, lesión o enfermedad bucal, y excluir enfermedades metabólicas o endócrinas.

Se eligió colectar muestras de saliva, por su naturaleza no invasiva, lo que no genera estrés en los niños, favoreciendo a la obtención de la autorización parental y cooperación del niño; además de ser fácil de obtener, y porque no necesita especial entrenamiento para el evaluador (Shariat,
Kargarfard, Tamrin, Danaee \& Karimi, 2014). Otra razón es la independencia de la tasa de flujo, ya que el cortisol salival representa la fracción biológicamente activa y libre de cortisol en sangre en respuesta al ejercicio (Gozansky, Lynn, Laudenslager \& Kohrt, 2005; Papacosta \& Nassis, 2011); y es una técnica de las más usadas actualmente (Silva, Mallozi \& Ferrari, 2007).

La recolección de datos se realizó en dos momentos. El primero fue tres semanas previas a la competición, en el que se colectó una muestra de cortisol salival (Toma 1) durante un día normal de entrenamiento en la semana, el cual mantuvo relativamente estable el volumen y la intensidad de la carga de entrenamiento (periodo sin situaciones estresantes relativas a la competición). Esta fue la línea base.

Considerando que varios investigadores definen el periodo precompetitivo como las 24 horas previas a la competición (Kivlighan et al., 2005), el segundo momento fue 16 horas antes de una competición que formaba parte del circuito de selección para el campeonato binacional de natación 2018 (dado que no era posible colectar las muestras durante la competición); en este segundo momento se aplicó el CSAI2R, y se colectó la segunda muestra de cortisol salival (Toma 2). Ambas tomas (Toma 1 y Toma 2), fueron efectuadas a las 16 horas dentro el recinto deportivo, y después del calentamiento que comprendió ejercicio aeróbico y estiramientos de los mayores grupos musculares.

\section{Colección de saliva}

Las dos recolecciones de muestras de cortisol salival se realizaron tomando en cuenta los criterios establecidos por el Manual Guía de Colección de Saliva de Salimetrics (Salimetrics, 2015). Antes de la toma de muestras de saliva, se solicitó a los participantes no consumir alimentos $60 \mathrm{mi}-$ nutos previos a la recolección de la muestra, y no lavarse o cepillarse los dientes 3 horas antes de la recolección. La técnica de expectoración pasiva, para minimizar posible contaminación, consistió en que los sujetos deben enjuagarse la boca con agua destilada estéril durante 10 segundos y posteriormente evacuar el contenido; se les pidió a los participantes esperar por 10 minutos sin hablar, esto para que el agua que utilizaron no diluya la saliva. Posteriormente se les indicó a los sujetos permanecer relajados y sentarse con su cabeza inclinada hacia el frente tratando de recolectar la mayor saliva posible en el área sublingual, esto durante dos minutos sin hacer ninguna clase de estímulo, y realizando el mínimo movimiento orofacial posible. Al término de los 2 minutos, se les pidió a los sujetos depositar la saliva recolectada en los tubos estériles recolectores de saliva. Todas las muestras de saliva aparecían claras sin color visible. Se recolectaron de cada sujeto de 1 a $2 \mathrm{ml}$ de saliva. Las muestras de saliva se almacenaron en los tubos estériles a $-80^{\circ} \mathrm{C}$ hasta su análisis.

\section{Ensayos}

Las concentraciones de cortisol salival se midieron usando ensayos inmunoabsorbentes ligados a enzimas (ELISA, Salimetrics ${ }^{\mathrm{TM}}$ ) por medio de un kit comercial (High Sensitivity Salivary Cortisol Enzyme Immunoassay Kit, Salimetrics 'CA, USA), realizando la curva de calibración para ajustar los resultados. La determinación se expresó en $\mu \mathrm{g} / \mathrm{dL}$. 
El flujo de saliva se calculó con el cociente volumen (ml)/ tiempo de recogida (min). El flujo de saliva se determinó como un posible indicador del estado de hidratación (Walsh, Laing, Oliver, Montague, Walters \& Bilzon, 2004).

\section{Análisis de datos}

Los datos se expresan en media $(M)$ y desviación típica (DT). La distribución de los datos fue analizada por la prueba Shapiro-Wilk. Dada la ausencia de una distribución normal de los datos, se usaron pruebas no paramétricas. Los análisis comparativos de la concentración de cortisol salival entre tres semanas (Toma 1) y 16 horas previas a la competición (Toma 2) fueron analizados con la prueba de Wilcoxon. Para correlacionar los parámetros fisiológicos y psicológicos se usó el coeficiente de correlación de Spearman. Adicionalmente, para el contraste de la hipótesis se midió el tamaño del efecto (SE) mediante la d de Cohen, considerando los siguientes umbrales: 0.1 , cambio pequeño; 0.3 moderado; 0.5 grande; 0.7 muy grande; 0.9 extremadamente grande (Hopkins, Marshall, Batterham \& Hanin, 2009). Se consideró una d = 0.2 como mínimo cambio apreciable.

\section{Resultados}

Con base en los rangos de edad estimados por Salimetrics (2015), para el primer grupo de edad (de 10 a 11 años), los valores medios de la concentración de cortisol salival en dicha submuestra, tanto en la línea base (Toma 1), como en el momento previo a la competición (Toma 2), se encontraron dentro del rango normal diurno $(<0.21 \mu \mathrm{g} / \mathrm{dL})$ (Tabla 1$)$.

Dentro de la Tabla 1, al comparar la concentración de cortisol salival entre ambas tomas, los resultados indicaron que en las muestras no hubo diferencias significativas entre la línea base (Toma 1) y 16 horas previas a la competición (Toma 2). Sin embargo, el tamaño del efecto mostró cambios relevantes entre las dos tomas, con un efecto extremadamente grande $(\mathrm{d}=-1.07 ; \mathrm{r}=-.47)$

Respecto a la ansiedad precompetitiva, basados en la puntuación media nominal de la escala de respuesta del instrumento, esta submuestra demostró moderada ansiedad somática, baja ansiedad cognitiva, y alta autoconfianza (Tabla 2). Finalmente, el nivel de cortisol salival tomado 16 horas previas a la competición, no correlacionó significativamente con alguna de las ansiedades (i.e. somática y cognitiva), ni con la autoconfianza precompetitivas.

En cuanto a la submuestra de 12 a 16 años de edad, los valores medios de cortisol salival, tanto en la línea base como en el momento precompetitivo, se situaron dentro del rango normal diurno $(<0.25 \mu \mathrm{g} / \mathrm{dL})$, excepto dos de los participantes. Al comparar la concentración de cortisol salival entre la línea base y el momento precompetitivo, los resultados mostraron que no hubo diferencia significativa entre las muestras (Tabla 1). Lo que se confirmó a través del tamaño del efecto, ya que no mostró cambios relevantes entre ambas tomas, obteniendo un efecto pequeño $(d=-0.13 ; r=-.06)$.

Con respecto a la ansiedad precompetitiva, basados en la puntuación media nominal de la escala de respuesta del instrumento, la segunda submuestra demostró baja ansiedad somática y cognitiva, y moderada autoconfianza (Tabla 2). Finalmente, el nivel de cortisol salival tomado 16 horas previas a la competición, no correlacionó significativamente con alguna de las ansiedades (i.e. somática y cognitiva), ni con la autoconfianza precompetitivas.

\begin{tabular}{|c|c|c|c|c|c|c|}
\hline & \multicolumn{2}{|c|}{ Toma 1} & \multicolumn{2}{|c|}{ Toma 2} & \multirow{2}{*}{ W } & \multirow{2}{*}{$p$} \\
\hline & $M$ & $D T$ & $M$ & $D T$ & & \\
\hline Submuestra $1(n=4)$ & & & & & & \\
\hline $\begin{array}{l}\text { Cortisol salival } \\
\text { Submuestra } 2(n=15)\end{array}$ & $0.11 \mu \mathrm{g} / \mathrm{dL}$ & 0.06 & $0.18 \mu \mathrm{g} / \mathrm{dL}$ & 0.07 & 0 & .14 \\
\hline Cortisol salival & $0.10 \mu \mathrm{g} / \mathrm{dL}$ & 0.04 & $0.11 \mu \mathrm{g} / \mathrm{dL}$ & 0.10 & 0 & .65 \\
\hline \multicolumn{7}{|c|}{$\begin{array}{l}\text { Tabla } 2 . \\
\text { Descriptivos de las variables psicológicas }\end{array}$} \\
\hline & \multicolumn{2}{|c|}{ Submuestra $1(n=4)$} & \multicolumn{4}{|c|}{ Submuestra $2(n=15)$} \\
\hline & $M$ & $D T$ & & $M$ & & \\
\hline Ansiedad cognitiva & 1.79 & 0.80 & & 2.20 & & \\
\hline Ansiedad somática & 1.85 & 1.01 & & 1.67 & & \\
\hline Autoconfianza & 2.12 & 0.61 & & 3.50 & & \\
\hline
\end{tabular}

\section{Discusión}

Con el objetivo de medir los niveles de ansiedad precompetitiva y su relación con la secreción de cortisol medido a nivel salival dentro de una competición oficial de natación., se llevó a cabo este trabajo.

Los resultados rechazan parcialmente las hipótesis planteadas. Primero, los niveles de cortisol salival precompetitivo aumentaron moderadamente con respecto a un día normal de entrenamiento en ambas submuestras; sin embargo, las diferencias de la concentración de cortisol salival entre ambos momentos (línea base vs. precompetición) no fueron significativas, concordando con otros estudios (e.g. Filaire et al., 2009; Le Panse et al., 2012) que trabajaron con muestras de edad similar a la nuestra.

No obstante, en el contraste de la hipótesis, el mínimo cambio apreciable de cortisol salival, indica cambio relevante en la submuestra de 10 y 11 años de edad, con tamaño del efecto extremadamente grande. Esto puede deberse a que, a menor edad suele haber menos experiencia competitiva, lo que concuerda de cierta manera con Kivlighan et al. (2005) quienes mostraron mayores cambios en cortisol salival en el grupo de novatos.

El incremento en concentración de cortisol salival, confirma previos reportes acerca de la respuesta anticipatoria de cortisol salival en competición deportiva. Esto es, que el cortisol antes de la competición refleja un mecanismo psicofisiológico, influenciado en parte por la anticipación cognitiva, que prepara al organismo para afrontar la competición. En este caso, sugiere adecuada respuesta al cambio (Eubank, Collins, Lovell, Dorling \& Talbot, 1997), ya que moderado incremento de cortisol ayuda al deportista en su competición, preparando al acuerpo para la actividad física, por ejemplo, moviendo sangre de las extremidades a los músculos grandes, afectando positivamente la memoria y las emociones que son importantes en el desempeño eficaz (Levine, 2000; Stansbury \& Gunnar, 1994).

No obstante, el mínimo cambio apreciable de cortisol salival no se constata para nadadores de 12 a 16 años de edad. Dicho resultado se opone a estudios que sí encontraron diferencias significativas en la concentración de cortisol salival entre la línea base, y previo a la competición (e.g. Elloumi et al., 2008; Filaire et al., 2001,2009 y 2010; Lautenbach et al., 2015; Maso et al., 2002; Reynoso-Sánchez et al., 2017). Esto puede obedecer a que las diferencias en cortisol tienden a incrementarse en deportistas de alto rendimiento 
(Cunniffe, Morgan, Baker, Cardinale \& Davies, 2015; Lautenbach et al., 2015), y no en deportistas amateur, como lo fue nuestra muestra.

Otra explicación a la discrepancia de los resultados aquí obtenidos, puede deberse a diferencias en el diseño del presente estudio con respecto a otros estudios ya citados. Por ejemplo, nosotros situamos el momento precompetitivo 16 horas antes de la competición, mientras que otros estudios lo situaron una hora antes de la competición (e.g. Maso et al., 2002). Nosotros consideramos la línea base en un día habitual de entrenamiento, en tanto que otros estudios la consideraron en un día de descanso (e.g. Elloumi et al., 2008; Filaire et al., 2001; Maso et al., 2002), o 24 horas antes de la competición (e.g. Bateup et al., 2002). Nosotros medimos el cortisol salival en la misma hora, mientras que en otro estudio se midió el cortisol salival en dos momentos a diferente horario (e.g. Filaire et al., 2010).

En cuanto a la intensidad de ansiedad precompetitiva, medida con el CSAI-2R, los resultados descriptivos denotan que las dos submuestras de estudio manifiestan bajos síntomas de ansiedad somática precompetitiva, como tensión muscular, nerviosismo o palpitaciones; al tiempo que tienen alta creencia de que pueden superar el reto de la competición. En este sentido, la autoconfianza pudo contribuir a que los niveles de cortisol salival no reflejaran estrés, ya que la autoconfianza puede moderar los efectos de ansiedad cognitiva y somática previas a la competición (Hardy, 1990); porque las personas con alta autoconfianza tienden a ver las situaciones estresantes como un reto (Dienstbier, 1989).

En tercer lugar, se rechaza la correlación entre la concentración de cortisol salival y las dimensiones de ansiedad precompetitiva, coincidiendo con otros estudios que se llevaron a cabo con diferentes deportistas, de ambos sexos, y tanto en adolescentes como en adultos (Bello, 2017; Carré et al., 2000; Haneishi et al., 2007; Mckay et al., 1997; Salvador et al., 2003; Thatcher et al., 2004). Esto puede significar que los nadadores no consideraron tal competición como potencialmente peligrosa, luego entonces, la poca variación de cortisol no denota elevada ansiedad. Lo anterior apoya que la asociación entre la respuesta hormonal anticipatoria y las dimensiones de ansiedad precompetitiva no es clara (Salvador et al., 2003). Además, sugiere que no es una asociación bidireccional, en cambio es altamente dependiente de diferencias individuales de la percepción social, previa experiencia, propensión para conductas específicas, entre otros, por lo que futuros estudios son necesarios para abordar estas variables.

Fisiológicamente, la concentración de cortisol salival se encuentra en niveles normales diurnos, al tiempo que se presentaron bajos síntomas somáticos y cognitivos de ansiedad antes de la competición, por lo que se corrobora un bajo nivel de estrés fisiológico y psicológico generado por la competición; esto posibilita mantener elevada la autoconfianza y una buena capacidad de atención-concentración durante la competición (González, Valdivia-Moral, Cachón, Zurita \& Romero-Ramos, 2017), lo que beneficiaría el rendimiento deportivo. Sin embargo, Núñez y García (2017) señalan que no existen evidencias empíricas o experimentales suficientes para esclarecer la relación entre ansiedad y rendimiento deportivo.
Algunos factores pudieron favorecer a no generar ansiedad precompetitiva. Por ejemplo, como lo sugieren Arruda et al. (2017), la posibilidad de percibir la competición como fácil; o bien interpretar la ansiedad como facilitadora (Jones \& Swain, 1992), ya que la ansiedad también depende de factores de personalidad, que orientan la interpretación cognitiva de la situación, lo que puede ser más importante para la respuesta hormonal.

Entre los hallazgos, se identificaron dos nadadores con una concentración de cortisol salival diurna por encima de los valores normales, sin embargo, su reporte de ansiedad, a través del inventario, refleja baja ansiedad somática y cognitiva. Entonces el aumento en cortisol salival podría deberse a variables extrañas como lo han señalado otros autores, tales como el sobreentrenamiento (Coutts, Reaburn, Piva \& Murphy, 2007; Crofford et al., 2004; Meussen et al. 2013), trabajo exhaustivo (Fujiwara et al., 2004), depresión (Bao, Ji, Van Someren, Hofman, Liu \& Zhou, 2004), o procesos inflamatorios no declarados en la anamnesis. Ello refuerza la importancia de estudiar factores fisiológicos y psicológicos en el entrenamiento deportivo.

Este estudio ha medido el cortisol salival y la ansiedad previo a una situación real de competición. Además, el cortisol salival se midió a la misma hora del día, lo que ayudó al mejor control de la variación diurna hormonal. En suma, los valores obtenidos 16 horas previas a la competición fueron comparados con un día habitual de entrenamiento, y no con niveles basales o de descanso.

Con base en lo anterior, este estudio tiene implicaciones teóricas que consisten en apoyar que niveles normales de cortisol reflejan baja ansiedad previa a una competición deportiva, y sugiere que dichos niveles pueden ser soportados por la alta autoconfianza. También tiene implicaciones prácticas a partir de una visión sobre estrés fisiológico y psicológico en pre competición de natación. Así, se apoya el utilizar mediciones hormonales junto con autoinformes psicológicos que pueden proveer una aproximación a examinar cambios en ansiedad y su relación con el rendimiento. Refuerza que es importante analizar la respuesta hormonal en el deporte como control del entrenamiento, así como para evaluar el potencial del deportista (Crewther, Heke \& Keogh, 2011), lo que asegure un buen programa de entrenamiento, y conduzca a un mejor rendimiento o mantenerlo. El utilizar técnicas mínimamente invasivas para el deportista puede ayudar a monitorear el estrés con frecuencia y en aproximación la competición.

Sin embargo, presenta limitaciones como el reducido tamaño muestral, inherente a este tipo de estudios, por lo que los resultados no pueden generalizarse. Así como la falta de control de otras variables, como factores estresantes externos (e.g. problemas familiares o problemas de sueño); o que la línea base no fue en un día de reposo total. Otra limitación es que no se consideró el resultado de la competición, por lo que sería importante contrastar niveles de cortisol con el resultado obtenido en la competición.

Futuros estudios podrían incorporar la medición de la ansiedad rasgo en la línea base, así como la dirección de ansiedad precompetitiva, ya que Jones y Swain (1992) sugieren que el impacto de la ansiedad puede obedecer a la interpretación que le da el deportista como facilitadora o 
debilitadora. Entonces la interpretación de los síntomas de ansiedad puede asociarse a las respuestas neuroendócrinas.

En conclusión, con esta muestra de nadadores, el análisis estadístico convencional por hipótesis nula, rechaza diferencias significativas en la concentración de cortisol salival entre las dos tomas para ambas submuestras. No obstante, el cambio mínimo apreciable confirma un cambio relevante de cortisol salival precompetitivo en nadadores de 10 y 11 años de edad. La muestra en general presenta bajo estrés fisiológico y psicológico previo a la competición, ello evidenciado tanto a través de un indicador fisiológico hormonal, en este caso el cortisol salival, como de un inventario psicológico. En este estudio, los factores de ansiedad somática y ansiedad cognitiva no se correlacionan con la concentración de cortisol 16 horas antes de la competición.

\section{Referencias}

Arruda, A.F., Aoki, M.S., Paludo, A.C., \& Moreira, A. (2017). Salivary steroid response and competitive anxiety in elite basketball players: Effect of opponent level. Physiology and Behavior, 177, 291-296. https://doi.org/10.1016/ j.physbeh.2017.05.017

Bao,A.M., Ji, Y.F., Van Someren, E.J., Hofman, M.A., Liu, R.Y., \& Zhou, J.N. (2004). Diurnal rhythms of free estradiol and cortisol during the normal menstrual cycle in women with major depression. Hormones and Behavior, 45, 93-102. https:// doi.org/10.1016/j.yhbeh.2003.09.004

Bateup, H.S., Booth, A., Shirtcliff, E.A., \& Granger, D.A. (2002). Testosterone, cortisol, and women's competition. Evolution and Human Behavior, 23, 181-192. https://doi.org/10.1016/ S1090-5138(01)00100-3

Bello, O.A. (2017). Variabilidad de la frecuencia cardiaca, cortisol salival y respuesta de ansiedad pre-entrenamiento y pre-competición: correlación test CSAI-2 (Tesis de grado). Recuperada de la base de datos de la Universidad de Ciencias Aplicadas y Ambientales. (2018).

Carré, J., Muir, C., Belanger, J., \& Putnam, S.K. (2006). Precompetition hormonal and psychological levels of elite hockey players: Relationship to the 'home advantage'. Physiology \& Behavior, 89, 392-398. https://doi.org/10.1016/ j.physbeh.2006.07.011

Coutts, A., Reaburn, P., Piva, T., \& Rowsell, G. (2007). Monitoring for overreaching in rugby league players. European Journal of Applied Physiology, 99(3), 313-324.

Crewther, B.T., Heke, T., \& Keogh, J.W. T (2011). The effects of training volume and competition on the salivary cortisol concentrations of Olympic weightlifters. Journal of Strength and Conditioning Research, 25, 10-15. http://dx.doi.org/ 10.1519/JSC.0b013e3181fb47f5.

Crofford, L.J., Young, E.A., Engleberg, N.C., Korszun, A., Brucksch, C.B., McClure, L.A., ..., \& Demitrack, M.A. (2004). Basal circadian and pulsatile ACTH and cortisol secretion in patients with fibromyalgia and/or chronic fatigue syndrome. Brain, Behavior, and Immunity, 18(4), 314-25. https://doi.org/ 10.1016/j.bbi.2003.12.011

Cunniffe, B., Morgan, K.A., Baker, J.S., Cardinale, M., \& Davies, B. (2015). Home versus away competition: Effect on psychophysiological variables in elite rugby union. International Journal of Sports Physiology and Performance, 10(6), 687694. http://dx.doi.org/10.1123/ijspp.2014-0370

Dickerson, S.S., \& Kemeny, M.E. (2004). Acute stressors and cortisol responses: a theoretical integration and synthesis of laboratory research. Psychological Bulletin, 130, 355-39., http:/ /dx.doi.org/10.1037/0033-2909.130.3.355

Dienstbier, R. A. (1989). Arousal and physiological toughness: implications for mental and physical health. Psychological Review, 96(1), 84-100.

Doan, B.K., Newton, R.U., Kraemer, W.J., Kwon, Y-H., \& Scheet, T.P. (2007). Salivary cortisol, testosterone, and T/C ratio responses during a 36-hole golf competition. International Journal of Sports Medicine, 28(6), 470-479. https://dx.doi.org/ 10.1055/s-2006-924557

Edwards, D.A., \& Kurlander, L.S. (2010). Women’s intercollegiate volleyball and tennis: Effects of warm-up, competition, and practice on saliva levels of cortisol and testosterone. Hormones and Behavior, 58(4), 606-613. https://doi.org/10.1016/ j.yhbeh.2010.06.015

Elloumi, M., Ounis, O.B., Tabka, Z., Van Praagh, E., Michaux, O., \& Lac, G. (2008). Psychoendocrine and physical performance responses in male Tunisian rugby players during an international competitive season. Aggressive Behavior: Official Journal of the International Society for Research on Aggression, 34(6), 623-632. https://doi.org/10.1002/ab.20276

Eubank, M., Collins, D., Lovell, G., Dorling, D., \& Talbot, S. (1997). Individual differences in pre-competition anxiety and hormonal concentration. Personality and Individual Differences, 23, 1031-1039.

Filaire, E., Alix, D., Ferrand, C., \& Verger, M. (2009). Psychophysiological stress in tennis players during the first single match of a tournament. Psychoneuroendocrinology, 34(1), 150-157. https://doi.org/10.1016/j.psyneuen.2008.08.022

Filaire, E., Maso, F. Sagnol, M., Ferrand, C., \& Lac, G. (2001). Anxiety, hormonal responses, and coping during a judo competition. Aggressive Behavior, 27, 55-63.

Filaire, F., Portier, H., Onena, N., \& Filaire, M. (2010). Réponses physiologiques et profil nutritionnel chez des adolescents lors d'un tournoi de tennis. Science \& Sports 25, 55-60. https:// doi.org/10.1016/j.scispo.2009.11.001

Fujiwara, K., Tsukishima, E., Kasai, S., Masuchi, A., Tsutsumi, A., Kawakami, N., . ., \& Reiko, K. (2004). Urinary catecholamines and salivary cortisol on workdays and days off in relation to job strain among female health care providers. Scandinavian Journal of Work, Environment and Health, 30(2), 129-38. http:/ /dx.doi.org/10.5271/sjweh.770

Gaab, J., Rohlede, N., Nater, U.M., \& Ehlert, U. (2005). Psychological determinants of the cortisol stress response: the role of anticipatory cognitive appraisal. Psychoneuroendocrinology 30, 599-610. https://doi.org/ 10.1016/j.psyneuen.2005.02.001

García, A., Martí, O., Vallès, A., Dal-Zotto, S., \& Armario, A. (2000). Recovery of the hypothalamic-pituitary-adrenal response to stress. Effect of stress intensity, stress duration and previous stress exposure. Neuroendocrinology, 72, 11425.

Gimeno, F., Buceta, J.M., \& Llantada, M.C.P. (2007). Influencia de las variables psicológicas en el deporte de competición: evaluación mediante el cuestionario Características Psicológicas Relacionadas con el Rendimiento Deportivo. Psicothema, 19, 667-672.

González, G., Valdivia-Moral, P., Cachón, J., Zurita, F., \& Romero-Ramos, O. (2017). Influencia del control del estrés en el rendimiento deportivo: la autoconfianza, la ansiedad y la concentración en deportistas. Retos, 32, 3-6.

Gozansky, W.S., Lynn, J.S., Laudenslager, M.L., \& Kohrt, W.M. (2005). Salivary cortisol determined by enzyme immunoassay is preferable to serum total cortisol for assessment of dynamic 
hypothalamic-pituitary-adrenal axis activity. Clinical Endocrinology, 63, 336-41. https://doi.org/10.1111/j.13652265.2005.02349.x

Haneishi, K., Fry, A.C., Moore, C.A., Schilling, B.K., Li, Y., \& Fry, M.D. (2007). Cortisol and stress responses during a game and practice in female collegiate soccer players. Journal of Strength \& Conditioning Research, 21, 583-588. http://dx.doi.org/ 10.1519/R-20496.1.

Hardy, L. (1990). A catastrophe model of performance in sport. En G. Jones y L. Hardy (Eds.), Stress and Performance in Sport (pp. 81-106). Chichester, UK: Wiley.

Hopkins, W.G., Marshall, S.W., Batterham, A.M., \& Hanin, J. (2009). Progressive statistics for studies in sports medicine and exercise science. Medicine and Science in Sports and Exercise, 41(1), 3-13. http://doi.org/10.1249/ MSS.0b013e31818cb278

Jaenes, J.C. (2000). Estado emocional y conducta deportiva: Ansiedad competitiva en corredores de maratón(Tesis doctoral). Universidad de Sevilla. España.

Jones, G., \& Swain, A. (1992). Intensity and direction as dimensions of competitive state anxiety and relationships with competitiveness. Perceptual and Motor Skills, 74, 467-472. https://doi.org/10.2466/pms.1992.74.2.467

Kivlighan, K.T., Granger, D.A., \& Booth, A. (2005). Gender differences in testosterone and cortisol response to competition. Psychoneuroendocrinology, 30, 58-71.https://doi.org/10.1016/ j.psyneuen.2004.05.009

Lautenbach, F., Laborde, S., Klämpf, M., \& Achtzehn, S. (2015). A link between cortisol and performance: An exploratory case study of a tennis match. International Journal of Psychophysiology, 98(2), 167-173. https://doi.org/10.1016/ j.ijpsycho.2015.10.002

Le Panse, B., Labsy, Z., Baillot, A., Vibarel-Rebot, N., Parage, G, Albrings, D., ..., \& Collomp, K. (2012). Changes in steroid hormones during an international powerlifting competition. Steroids, 77, 1339-1344. https://doi.org/10.1016/ j.steroids.2012.07.015

Levine, S. (2000). Influence of psychological variables on the activity of the hypothalamic-pituitary-adrenal axis. European Journal of Pharmacology, 405, 149-160. https://doi.org/10.1016/S00142999(00)00548-3

Loucks, A.B., Mortola, J.F., Girton, L., \& Yen, S.S. (1989). Alterations in the hypothalamic-pituitaryovarian and the hypothalamic-pituitary-adrenal axes in athletic women. Journal of Clinical Endocrinology and Metabolism, 68(2), 402-11. https://doi.org/10.1210/jcem-68-2-402

Martens, R., Vealey, R.S., \& Burton, D. (1990). Competitive anxiety in sport. Champaing, IL: Human kinetics.

Maso, F., Cazorla, G., Godemet, M., Michaux, O., Lac, G., \& Robert, A. (2002). Influence d'une compétition de rugby sur le taux de cortisol salivaire Influence of an international rugby mah upon the concentration of salivary cortisol. Science et Sports, 17, 302-305.

McKay, J.M., Selig, S.E., Carlson, J.S., \& Morris, T. (1997). Psychophysiological stress in elite golfers during practice and competition. Australian Journal of Science and Medicine in Sport, 29, 55-61.

Meeusen, R., Duclos, M., Foster, C., Fry, A., Gleeson, M., Nieman, D., ..., \& Urhausen, A. (2013). Prevention, diagnosis, and treatment of the overtraining syndrome: joint consensus statement of the European College of Sport Science and the American College of Sports Medicine. Medicine and Science in Sports and Exercise, 45(1), 186-205.

Núñez, A., \& García,A. (2017). Relación entre el rendimiento y la ansiedad en el deporte: una revisión sistemática. Retos: nuevas tendencias en educación física, deporte y recreación, (32), 172177.

Papacosta, E., \& Nassis, G.P. (2011). Saliva as a tool for monitoring steroid, peptide and immune markers in sport and exercise science. Journal of Science and Medicine in Sport, 14, 424434.

Papacosta, E., Nassis, G.P., \& Gleeson, M. (2016). Salivary hormones and anxiety in winners and losers of an international judo competition. Journal of Sports Sciences, 34, 1281-1287.

Pineda-Espejel, H.A., López-Walle, J.M., \& Tomás, I. (2014). Validación de la versión mexicana del CSAI-2R en sus escalas de intensidad y dirección. Revista Mexicana de Psicología, 31(2), 198-212.

Ponseti, F.J., García, A., Cantallops, J., \& Vidal, J. (2017). Diferencias de sexo respecto de la ansiedad asociada a la competición deportiva. Retos: nuevas tendencias en educación física, deporte y recreación, (31), 193-196.

Reynoso-Sánchez, L.F., Hoyos Flores, J.R., García-Dávila, M., Rosas Taraco, A.G., Jaenes Sánchez, J. C., López-Walle, J. M., \& Hernández-Cruz, G. (2017). Cortisol y estrés-recuperación durante un periodo competitivo en jugadores de balonmano. Revista de Psicología del Deporte, 26(2), 125-131.

Salimetrics Saliva Collection and Handling Advice data revisited. (n.d.). Retrieved march 8, 2015, from the Trust SalivaBio Collection Methods From Salimetrics; https:// www.salimetrics.com/assets/documents / Saliva_Collection_Handbook.pdf

Salvador, A., Suay, F., González-Bono, E., \& Serrano, M.A. (2003). Anticipatory cortisol, testosterone and psychological responses to judo competition in young men. Psychoneuroendocrinology, 28, 364-375.

Shariat, A., Kargarfard, M., Tamrin, S.B.M., Danaee, M., \& Karimi, H. (2014). Strength-training and biological rhythm of male sex hormone among judoists. Biological Rhythm Research, 45(4), 625-631.

Silva, M.L., Mallozi, M.C., \& Ferrari, G.F. (2007). Salivary cortisol to assess the hypothalamic-pituitary-adrenal axis in healthy children under 3 years old. Jornal de Pediatria, 83(2), 121126.

Stansbury, K., \& Gunnar, M.R. (1994). Adrenocortical activity and emotion regulation, En Fox, N. (Ed.), Monographs for the Society of Research in Child Development, vol. 59 (pp. 108134).

Steinacker, J.M., Lormes, W., Kellmann, M., Liu, Y., Reißnecker, S., Opitz-Gress, B. et al. (2000). Training of junior rowers before world championships. Effects on performance, mood state and selected hormonal and metabolic responses. Journal of Sports Medicine and Physical Fitness, 40, 327-335.

Tamorri, S., \& Benzi, M. (1990). Psicologia dello sport. En Silvij S. Medicina dello sport. SEU, Roma.

Thatcher, J., Thatcher, R., \& Dorling, D. (2004). Gender differences in the pre-competition temporal patterning of anxiety and hormonal responses. Journal of Sports Medicine and Physical Fitness, 44, 300-308.

Valdés, H.M. (2002). La preparación psicológica del deportista. Mente y rendimiento humano. Barcelona: INDE.

Walsh, N.P., Laing, S.J., Oliver, S.J., Montague, J.C., Walters, R., \& Bilzon, J.L.J.(2004). Saliva parameters as potential indices of hydration status during acute dehydration. Medicine and Science in Sports and Exercise, 36(9), 1535-1542.

Weinberg, R. S., \& Gould, D. (2010). Fundamentos de psicología del deporte y el ejercicio físico. Barcelona: Ariel. 